Fig. 1 und 2: Blüte von hinten gesehen (rückwärts das Tragblatt des Ährchens, vorne das Vorblatt.

Fig. 3 und 4: Vorblätter (von schon fruchtenden Ährchen).

Fig. 5 und 6: Tragblätter (von schon fruchtenden Ährchen).

\title{
Beiträge zur Kenntnis der Gattung Crepis.
}

\section{Von Dr. Josef Stadlmann.}

(Aus dem botanischen Institute der k. k. Universität Wien.)

(Mit Tafel XI.)

Auf den zwei botanischen Reisen, welche im Jahre 1904 und 1907 vom Naturwissenschaftlichen Vereine an der Universität Wien nach Bosnien veranstaltet wurden, fanden die Teilnehmer beide Male an getrennten Standorten eine Crepis-Art, welche das erstemal $^{1}$ ) als Crepis pannonica in die Aufzählung der gefundenen Pflanzen aufgenommen wurde, sich das zweitemal doch als eine andere Pflanze erwies, da mehr Material zur Verfügung stand. Einen wertrollen Fingerzeig für die Identifizierung der Pflanze erhielten wir dann am Sehlusse der zweiten Reise, als uns Herr K. Maly im bosnisch-herzegowinischen Landesmuseum das Herbarexemplar des seit der Entdeckung verschollenen Mulgedium (Crepis) Blavii zur Ansicht vorlegte und wir sofort die Ähnlichkeit unserer Pflanze mit dem Blauschen Original erkannten. Herr Maly war so liebenswürdig, dieses Original mir zum Vergleiche und zur Untersuchung zu übersenden, deren Ergebnisse im folgenden dargelegt werden sollen.

Crepis Blavii Ascherson, Zeitschrift der Ges. f. Erd̄kunde zu Berlin, 1870, p. 549; Blau, Reisen in Bosnien etc., 1877, p. 81 (ohne Beschreibung). Mulgedium Blavii A scherson in Ascherson et Kanitz, Cat. corm. et anthophyt. Serb. etc., 1877, p. 41, nr. 1118 (ohne Beschreibung); Bot. Ztg., 1879, p. 260 (mit Beschreibung). Crepis rigida Visiani, Fl. Dalm., 1847, II., p. 119 [non W. K.]. Crepis pannonica Stadlmann in Österr. botan. Zeitschr. 1906, p. 271 [non (Jacqu.) C. Ko ch]. Planta perennis omnibus partibus lactescens. Radix simplex recta profundeque descendens, calamum aut digitum crassitie aequans, extus fusca, interdum squamis foliorum emortuorum contecta, plurimum unum eaulem rarius duos vel tres proferens. Caules $50-80 \mathrm{~cm}$ alti, saepius etiam altiores, erecti diametro 2-5 mm, firmi, rigidi, obtuse angulati, basi purpurascentes, vi s cos i glanduloso-villosissimi, superne sulcati, minus villosi vel summi paene glabri, paullulum flexuosi, ad inflorescentiam composito-racemosam usque foliati, plures ramos floriferos proferentes, saepe etiam ramiferi in longitudinem $20-30 \mathrm{~cm}$; rami axillares

1) Österr. botan. Zeitschr., 1906, p. 271. 
breves rigidi angulo acuto rarius recto prodeuntes inde areuatim erecti. Folia crassa, rigida, dentata, apice acuta, utrimque venosa, venis subtus eminentibus margine glandulosa, lamina utrimque parcius hirto-glandulosa; radicalia aut caulina infima ovata, in petiolum decurrentia, rarius subsessilia, postice sinuato-laciniata, versus apicem sinuato-dentata; caulina reliqua amplexicaulia, auriculis rotundatis, setulis brevissimis scabra, margine glandulosa, inferiora ovata, sinuato-dentata, superiora sagittata, sinuato-dentata, dentibus subrecurvis, raro integra, summa hastata nonnisi basin versus dentata, alia integerrima. Inflorescentia ramosa, ramorum capitula $1-5$ multiflora, pedunculi crassi, areuatim erecti, sulcati, bracteae subfrondosae, lanceolatae, acuminatae, suffultae, raro tenues, squamosae ; involucrum campanulato-cylindricum, 9-12 mm longum, flavo. viride, 10-14 folium, foliola longiora et breviora, lineari-oblonga, obtusa, margine parce cano-tomentosa, eglandulosa, apice sphacelata; corollae aureae, tubulus pallidus, ligula linearis, inaequaliter incisa, quadri- aut quinquefida. Semina (achaenia) $4-5.5 \mathrm{~mm}$ longa, sulcata, eylindrica non compressa (interdum compressa esse videntur), apice attenuata, fusca; pappus pilosus, sessilis, candidus, sine anulo pilorum tenuium, qui est in basi pappi generis Mulgedii, $8 \mathrm{~mm}$ longus.

Ar. geogr.: Bosnien: Straßenränder zwischen Han Prolog und Han Vaganj an der Straße Livno-Sinj, ca. $1000 \mathrm{~m}$, Kalk, lg. J. Stadlmann, F. Faltis, M. Hellweger, 20. VII. 1904 (vgl. Österr. botan. Zeitschr., l. c., p. 271, als Crepis pannonica); 'TušnicaGebiet: Südabhang des Vitrnjak mali gegenüber von Gradac westlich von Stipanié; zwischen Gebüsch, ca. 1200-1300 m, Kalk, lg. J. Stadlmann, F. Faltis, E. Wibiral, 17. VII. 1907. Dinarische Alpen: An Ackerrändern bei Crnilug, ca. $750 \mathrm{~m}$, Kalk, lg. E. Janchen, B. Watzl, 7. VII. 1907. Notiert von E. Janchen an der bosnisch-dalmatinischen Grenze bei Uništa an Karsthängen nördlich und westlich vom Orte (vgl. Österr. botan. Zeitschr., 1908, p. 361). Dalmatien: In agris et pascuis circa Glavase $^{1}$ ) ad radices montis Dinara et ad Polazza inter Knin et Vrlika (Visiani, Fl. Dalm., II., p. 119). Herzegowina: Habitat in fruticetis solo lapidoso inter Paljev-dol et Pakračic inter Nevesinje et Mostar, lg. Blau (vgl. Bot. Ztg., 1879, p. 260). Nach gütiger Mitteilung von Herrn K. Maly heißt die Lokalität auf der Spezialkarte Pakračuža und liegt etwa $800-1000 \mathrm{~m}$ hoch.

Crepis Blavii stellt eine vielfach verkannte Art dar. Der erste, welcher die Pflanze fand, war $\nabla$ isiani, der sie mit Crepis pannonica verwechselte. Von dieser unterscheidet sich unsere Pflanze durch den in die Länge gezogenen, infolge der kurzen, fast gleichlangen Äste, fast zylinderischen Blütenstand und durch die Drüsigkeit der Blätter und der unteren Stammteile. Cr. pannonica hat ebensträußige Blütenstände und nicht drüsig-klebrige Blätter,

1) Die Schreibart der Spezialkarte ist Glavae und Palača. 
die sich infolge der kurzen, steifen Behaarung sehr rauh anfühlen. Die Stelle, basi foliisque villosis" bei Visiani gibt eine Andeutung, dafs es sich vielleicht nicht um die pannonische Pflanze handelt. Die Standorte Glavac (Visiani) und Uništa (Janchen) sind etwa $5 \mathrm{~km}$ voneinander entfernt, so daß bei den dort herrschenden gleichen Vegetationsbedingungen der Schluß auf die Identität der Visianischen Pflanze mit Crepis Blavii wohl berechtigt ist.

Was nun den Vergleich unserer Pflanze mit dem Blauschen Original anbelangt, welches von Ascherson später als Mulgedium Blavii beschrieben wurde, so ist es wohl nur dem Mangel an geeignetem Untersuchungsmaterial zuzuschreiben, daß die Pflanze nicht als zur Gattung Crepis gehörig erkannt wurde. Infolge allzu starken Pressens dürften auch die noch unreifen Achänien ziemlich zusammengedrückt worden sein, so daß sie in ihrer flachen Form den Früchten von Mulgedium einigermaßen gleichen. Aber es fehlt der für Mulgedinm charakteristische feine Haarkranz am Grunde des Pappus, so daß nach den Früchten Blau's Pfianze sicher eine Crepis darstellt. Habituell ist wohl das Blausche Original auf keinen Fall einem Mulgedium ähnlich. Vielleicht war die starke Bedrüsung für die spätere Einreihung unter Mulgcdium ausschlaggebend, da sie auch in der Beschreibung an erster Stelle erwähnt ist. Das Original gleicht unserer Pflanze so vollständig, daß an der Identität beider Pflanzen kein Zweifel sein kann und somit das so lange verschollene Mulgedium Blavii aufgeklärt erscheint ${ }^{1}$ ).

Daß die Pflanze so lange nicht richtig beurteilt wurde, mag wohl in der Abgelegenheit aller Standorte mit einen Grund haben. Dazu ist ferner das Material Visiani's schwer zugänglich; Bl a u's Standort wurde zwar von Maly besucht, die Pfanze aber dort nicht gefunden. Die nunmehr bekannte Verbreitung deutet darauf hin, daß die Pflanze auch noch zwischen den äußersten Standorten (Dinarische Alpen und Gegend zwischen Nevesinje und Mostar) verbreiteter sein dürfte, nnd daß die Standorte am Prolog und an den Südausläufern der Tušnica nur zwei zufällig bekannte Punkte in dem ganzen Verbreitungsgebiete darstellen. Auch der pflanzengeographische Charakter der Standorte ist ziemlich der gleiche, Karstheide oder niedriger Buschwald im Karstgerölle in

1) Es dürfte vielleicht ganz gern gesehen werden, wenn hier die nicht überall leicht zugängliche Originalbeschreibung wiedergegeben wird; vgl. Bot. Ztg., 1879, p. 260: MIulgedium Blavii Aschs. Planta perennis, glanduloso-villosissima. Caulis (in exemplo unico putato) $0.39 \mathrm{~m}$ altus, ad inflorescentiam composito-racemosam usque foliatus. Folia firma, oblonga, acuta, subrepando. denticnlata, inferiora in petiolum brevem attenuata, reliqua sessilia, auriculis rotundatis amplexicaulia, summa diminuta, integerrima. Inflorescentiae rami capitula 1-3na multifiora gerentes, bracteis subfrondosis lanceolatis acuminatis suffulti. Involucri campanulato-cylindrici $0.012 \mathrm{~m}$ longi phylla inferiora 10-12na. lineari-oblonga, obtusa; corollae aureae; achaenium (immaturum) $0.0025 \mathrm{~m}$ longum; compressum, sursum subattenuatum utrinque 3-costatum; pappus candidus, $0.008 \mathrm{~m}$ longus. 
einer Seehöhe von $700-1200 \mathrm{~m}$ und gewöhnlich südliche Exposition.

Crepis Blavii dürfte nach dem Gesagten eine die $C r$. pannonica im Balkangebiete vertretende Art sein, während $\mathrm{Cr}$. pannonica sich in Ungarn, Siebenbürgen und vielleicht den angrenzenden Teilen von Rumänien und Rucland findet. Auch Exemplare von der Steppe bei Cherson (lg. Rehmann, Steppe bei Beszlearak, $\mathrm{H}$. Kerner) dürften zu $\mathrm{Cr}$. pannonica gehören, während ich das Vorkommen in Asien nach einem vorliegenden Exemplar von Regel aus Turkestan bezweifeln möchte. Wie schon die Varietätsangabe „var. Songarica K. K." andeutet, dürfte es sich wohl um eine ganz andere Art handeln.

Im Tušnicagebiete kommt am Standorte der Crepis Blavii auch $C r$. chondrilloides Jacqu., Enum. stirp. Vind., 1762, p. 312, vor. Zwischen diesen beiden Arten findet sich nun auch ihr Bastard, der im folgenden beschrieben werden soll.

Crepis Malyi Stadlmann = Crepis chondrilloides Jacqu. $\times$ Cr. Blavii Ascherson.

Planta inter parentes characteribus et altitudine plane intermedia. Differt a Cr. chondrilloide foliis non usque ad rhachin pinnatifidis, laciniis \pm latis, caulinis basi amplexicaulibus, auriculatis, ramis arcuatim erectis non rectangulari-patentibus unum vel duo capitula ferentibus; a $\mathrm{Cr}$. Blavii lamina foliorum pinnatifida, ramis unum raro duo capitula ferentibus; tota planta glanduloso-villosa usque ad duas partes altitudinis, foliola capitulorum latere dorsali cristate glandulosa.

B os n i en: Tušnicagebiet: Südabhang des Vitrnjak mali, gegenüber von Gradac, westlich von Stipanić; zwischen Gebüsch unter den Stammeltern; ca. 1200-1300 m, Kalk, lg. J. Stadlmann, F. Faltis, E. Wibiral, 17. VII. 1907. Im Gebiete der Dinarischen Alpen und am Prologpasse findet sich neben Crepis Blavii auch $C r$. chondrilloides, so das dieser Bastard auch noch anderwärts sicher gefunden werden dürfte.

Die vorliegende Pflanze, welche ich nach dem eifrigen Erforscher der bosnisch-herzegowinischen Flora, dem wir in einer Reihe von schwierigen Fällen bereitwilligste und weitgehendste Auskunft und Unterstützung sowohl bei der Feststellung des Reiseplanes, wie auch bei der vorliegenden Bearbeitung verdanken, Crepis Malyi benenne, stellt einen Bastard dar, welcher in geradezu idealer Weise die Mitte zwischen den habituell ziemlich verschiedenen Stammeltern hält und von ihnen leicht zu unterscheiden ist.

Die allgemeine Wuchsform besitzt Crepis Malyi von der Cr. Blavii, nämlich den steifen, aufrechten Stamm, die kurzen Blütenäste, die sofort schiefwinkelig oder etwas gekrümmt aufstreben. Ebenso erinnert das stellenweise Auftreten von 2-3 Blütenköpfchen an den Seitenästen an die vorgenannte Art. Die Blattform stellt ein Mittel dar zwischen dem bis zur Blattspreite fein geteilten Blatt von $C r$. chondrilloides und dem nur gezähnten, oft fast 
ganzrandigen Blatt von Cr. Blavii. Die hüher am Stamme stehenden Blätter sind stengelumfassend, wie die von Cr. Blavii. Die ganze Pflanze ist am Stamme und an den Blättern drüsenhaarig und klebrig wie die Stammeltern. Da $\mathrm{Cr}$. chondrilloides sehr stark in der Behaarung ynd Bedrüsung wechselt - es gibt Exemplare mit einfach flaumigen, kurzen Haaren, solche mit kurzen, drüsenlosen Borsten, und endlich solche mit reichlichen, langen Drüsenhaaren - so erscheint auch der Bastard einigermaßen variabel, indem bei einzelnen Exemplaren nur der untere Stengelteil mit den Blättern stark, aber kurzdrüsig ist, bei den anderen die ganze Pflanze einschließlich der Rückenseite der Köpfehenschuppen ziemlich gleichmäßig mit \pm langen Drüsenhaaren besetzt ist. An den Schuppen sind die Drüsen kürzer gestielt und bilden eine Art Kamm. Der Pollen ist nach den vorgenommenen Untersuchungen größtenteils fertil.

Die Tafel, welche links Crepis chondrilloides, rechts Crepis Blavii, in der Mitte deren Bastard Crepis Malyi im Maßstabe 1:4 darstellt, wurde nach einer Aufnahme angefertigt, die mir Frl. A. Mayer in liebenswürdigster Weise besorgte. Es sei ihr hiermit der beste Dank ausgesprochen.

\title{
Zur Nomenklatur des gemeinen Sonnenröschens.
}

\author{
Von E. Janchen (Wien). \\ (Mit 2 Textabbildungen.) \\ (Schluß. ${ }^{1}$ )
}

Ganz abgesehen davon, daß der Name Hcl. nummularizm vor allen anderen die Priorität hat, wäre es aber auch schwer, unter den jüngeren einen zu finden, der die Pflanze, um die es sich handelt, ebenso klar und einwandfrei bezeichnet. In Betracht kämen zunächst die folgenden Namen: Hel. Chamaccistus Miller, Hel. vulgare Gärtner, Hel. tomentosum S. F. Gray.

Helianthemum Chamaecistus Mille ${ }^{2}$ ) umfaßst sowohl Hel. hirsutum als auch Hel. nummularium und wurde auch stets im Sinne eines derartigen oder noch viel weiteren Sammelbegriffes von jenen Autoren angewendet, welche die hier unterschiedenen Arten nicht spezifisch trennen. Eine Präzisierung des Namens im Sinne von Hel. nummularium findet sich erst bei Simonkai ${ }^{s}$ ), eine Präzisierung im Sinne von Hel. hirsutum bei Rouy und Fou ca ud ${ }^{ \pm}$). Derartige Einschränkungen sind aber nicht nur etwas willkürlich, sondern sie haben auch den entschiedenen Nachteil, daß die Klar-

1) Vgl. Jahrg. 1908, Nr. 10, S. 406.

2) The Gard. Dict., ed 8 (1768), nr. 1 .

3) Enum. flor. Transsilv. (1886), pag. 106.

4) Flore de France, II (1895), pag. 295. 